\title{
Quo vadis, ambulante Medizin?
}

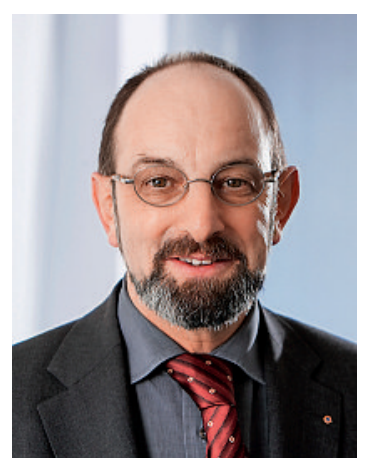

Die Diskussionen zur ambulanten Praxismedizin werden zur Zeit besonders intensiv geführt, auch wegen der Volksinitiative «Ja zur Hausarztmedizin». Gleichzeitig haben die Medien die Einkommensverhältnisse innerhalb der Ärzteschaft zum Anlass genommen, auf die angebliche Kluft zwischen Spezialisten und Grundversorgern hinzuweisen. Dabei bringen sie Zahlen ins Spiel, die so nicht vergleichbar sind: Die Absenkung der spezialärztlichen Einkommen zugunsten der Grundversorger zu fordern, klingt zwar gut, greift jedoch schlicht zu kurz. Diese Forderung vergisst, dass Spezialärzte ihre Einkommen meist nur zu einem kleinen Teil aus Umsätzen des KVG generieren, Grundversorger hingegen fast ausschliesslich. Der Trugschluss sei noch anderweitig belegt - wenn Spezialisten angeblich zu viel verdienen, zu welcher Gruppe werden dann die Psychiater gezählt?

\section{Zusätzliche Geldmittel sind für eine sachgerechte Tarifrevision nötig}

Diese Diskussionen beeinflussen auch unsere Verhandlungen zur Revision der Tarifstruktur TARMED mit santésuisse. Eines der Ziele ist die Besserstellung der Grundversorger, was sowohl bei der FMH als auch bei santésuisse unbestritten ist. Erhebliche Unterschiede zwischen den Verhandlungspartnern bestehen nach wie vor bei der Frage der Finanzierung. santésuisse will eine lineare Absenkung der Einkommen der Spezialärzte zugunsten der Grundversorger - was allein schon wegen der Vorgaben im KVG nicht möglich ist. Für die FMH ist klar, dass eine Revision der Tarifstruktur in Zusammenarbeit mit den betroffenen Fachgesellschaften und nach betriebswirtschaftlichen Kriterien erfolgen muss - so schreibt es auch Art. 43 KVG vor. Die Neuberechnung der Positionen mit Minutagen, Sparten und den angepassten Kostenmodellen sind zu plausibilisieren. Wir gehen davon aus, dass dabei möglicherweise Geldmittel frei werden, die aber im voraus nicht beziffert werden können und wohl auch nicht ausreichen. Es sind zusätzliche Volumina nötig, um eine sachgerechte Tarifrevision zu realisieren. Entsprechende Gespräche dazu finden auf höchster Ebene in den nächsten Wochen statt; die FMH nimmt die Revision der Tarifstruktur TARMED aber so oder so rasch in Angriff.

Als erstes positives Zeichen für die praktizierenden Ärzte hat santésuisse die Besuchs-Inkonvenienz-Pauschale (BIP) bis zum 31. Dezember 2010 verlängert, eine erfreuliche Botschaft, die für die ambulante Medizin hoffentlich Signalwirkung hat.

Die Revision der Analysenliste (AL) wirkt sich verheerender aus, als dies vom BAG vorausgesagt wurde: Grundversorger verzeichnen Labor-Umsatzverluste von gegen 30 Prozent. Damit werden die von der FMH vor Einführung des neuen Labortarifs gemachten Simulationen bestätigt. Das BAG will aber mit seinem Monitoring weder die sachgerechte Berechnung der Revision des Tarifs überprüfen, noch die Abgeltung der vermehrten Aufwendungen der Ärzteschaft für das Monitoring des BAG übernehmen. Zeichnet sich weiterhin kein Konsens für ein gemeinsames Monitoring der Auswirkungen der AL ab, wird die FMH unbeirrt ihr eigenes Monitoring durchführen und veröffentlichen. Damit verfolgen wir das Ziel, dass Korrekturen aufgrund einer betriebswirtschaftlichen Berechnung gemäss Vorgaben des KVG stattfinden. Wir haben schon früh mit dem «Point of Care-Modell» eine Alternative geschaffen, welche vom BAG bisher einfach ignoriert wurde.

Auch bei der ärztlichen Medikamentenabgabe (AMA) wurden mit der Absenkung der Marge und mit der Forderung nach der Abschaffung der AMA in der Revision des Heilmittelgesetzes (HMG) falsche Signale gesetzt. Diese schwächen die ambulante periphere Medizin zusätzlich. Wo bleiben die Anliegen der Patientinnen und Patienten, welche die Dienstleistung der AMA ausserordentlich schätzen, wie alle Umfragen klar zeigen? Die Ärzteschaft hat bei santésuisse bereits am 14. Dezember 2009 eine Projektskizze zur margenunabhängigen Abgeltung der AMA eingereicht, welche dem ewigen Vorwurf der «falschen Anreize» bei der ärztlichen Medikamentenabgabe, begegnen soll.

Im Mai 2010 werden wir dieses zukunftsweisende Projekt erstmals mit der santésuisse diskutieren.

\section{Die FMH schlägt eine margen- unabhängige Abgeltung der ärztlichen Medikamentenabgabe vor}

Informationen zum Projekt RFE (Reason for Encounter) der praktizierenden Ärzteschaft zum Erfassen des Nachfrageverhaltens auch im Hinblick auf die Einführung von SwissDRG 2012 finden Sie in dieser Ausgabe der Schweizerischen Ärztezeitung auf Seite 615. Beteiligen Sie sich an dieser Erhebung - sie ist freiwillig, kostenlos, einfach durchführbar und hilft uns in Tariffragen und -verhandlungen enorm weiter.

Dr. med. Ernst Gähler, Vizepräsident FMH, Verantwortlicher Ressort Tarife und Verträge 